DOI: 10.17516/1997-1397-2021-14-3-351-359

УДК 534.18

\title{
Effective Acoustic Equations for a Layered Material Described by the Fractional Kelvin-Voigt Model
}

\author{
Alexey S. Shamaev* \\ Vladlena V.Shumilova ${ }^{\dagger}$ \\ Ishlinsky Institute for Problems in Mechanics RAS \\ Moscow, Russian Federation
}

Received 10.12.2020, received in revised form 16.01.2021, accepted 05.03.2021

\begin{abstract}
The paper is devoted to the construction of effective acoustic equations for a two-phase layered viscoelastic material described by the Kelvin-Voigt model with fractional time derivatives. For this purpose, the theory of two-scale convergence and the Laplace transform with respect to time are used. It is shown that the effective equations are partial integro-differential equations with fractional time derivatives and fractional exponential convolution kernels. In order to find the coefficients and the convolution kernels of these equations, several auxiliary cell problems are formulated and solved.
\end{abstract}

Keywords: homogenization, acoustic equations, viscoelasticity, fractional Kelvin-Voigt model.

Citation: A.S. Shamaev, V.V. Shumilova, Effective Acoustic Equations for a Layered Material Described by the Fractional Kelvin-Voigt Model, J. Sib. Fed. Univ. Math. Phys., 2021, 14(3), 351-359.

DOI: 10.17516/1997-1397-2021-14-3-351-359.

The study of macroscopic acoustic behavior of heterogeneous viscoelastic materials with periodic microstructure is one of the most significant problems in acoustical engineering when dealing with polymer based composites. The most rigorous and widely accepted mathematical tool for the theoretical part of this study is the theory of homogenization. Using techniques of homogenization, the actual highly inhomogeneous periodic viscoelastic composite can be replaced by the corresponding effective (homogenized) material with the similar acoustic properties.

It is well known that short memory effects in microheterogeneous viscoelastic Kelvin-Voigt materials lead to the appearance of long memory effects in the corresponding effective media (see [1-3]). In other words the acoustic equations for these materials, which are partial differential equations, become partial integro-differential equations after homogenization. The same result was observed for two-phase materials, in which the first phase is an elastic material whilst the second one is a viscoelastic Kelvin-Voigt material $[4,5]$.

In recent years there has been an increasing number of papers devoted to the development of fractional models in viscoelasticity (see, for instance, [6-8] and the reference therein). Such models consist of differential or integro-differential equations with fractional derivatives. The growing popularity of fractional models is explained by their ability of describing the complex behaviour of viscoelastic materials using a small number of parameters.

In this paper, we consider a mathematical model describing small displacements of a twophase layered viscoelastic material whose behavior is described by the fractional Kelvin-Voigt model. This model consists of a system of partial differential equations with fractional time

\footnotetext{
*https://orcid.org/0000-0003-2766-6382

†v.v.shumilova@mail.ru https://orcid.org/0000-0003-3830-7924

(c) Siberian Federal University. All rights reserved
} 
derivatives and rapidly oscillating piecewise constant coefficients, conditions of ideal contact between layers, and homogeneous initial and outer boundary conditions. Using the two-scale convergence method $[9,10]$ and applying the Laplace transform, we show that the corresponding effective model involves a system of partial integro-differential equations with fractional time derivatives and constant coefficients. By solving a number of auxiliary cell problems, we calculate these coefficients and find that the integral parts of the effective equations are of convolution type and their kernels are fractional exponential Rabotnov's functions. Thus, we rigorously establish that long memory effects mentioned above also appear in the effective material that corresponds to the fractional Kelvin-Voigt material.

\section{Original acoustic equations}

Consider a bounded domain $\Omega=(0, L)^{3}$ occupied by two-phase viscoelastic material with a periodic microstructure. Let $\varepsilon \ll L$ be a small positive parameter characterizing the heterogeneity period of the viscoelastic material. We suppose that every phase is isotropic and consists of the union of layers that are parallel to the $\mathrm{Ox}_{2} x_{3}$ plane. More precisely, denote

$$
\begin{gathered}
D_{2 \varepsilon}=(0, L) \cap\left(\bigcup_{k=0}^{\infty}\left(\varepsilon\left(h_{1}+k\right), \varepsilon\left(h_{2}+k\right)\right)\right), \quad D_{1 \varepsilon}=(0, L) \backslash \bar{D}_{2 \varepsilon}, \\
h_{1}=\frac{1-h}{2}, \quad h_{2}=\frac{1+h}{2}, \quad 0<h<1
\end{gathered}
$$

and assume that the sets $\Omega_{1 \varepsilon}=D_{1 \varepsilon} \times(0, L)^{2}$ and $\Omega_{2 \varepsilon}=D_{2 \varepsilon} \times(0, L)^{2}$ are occupied by the first and the second phase, respectively.

Note that the periodicity cell $Y_{\varepsilon}$ of the above layered material may be extracted in different ways. For our convenience, we will assume that $Y_{\varepsilon}=\varepsilon Y$, where $Y=(0,1)^{3}$ is a unite cube. The cube $Y$ can be decomposed into two parts $Y_{1}$ and $Y_{2}$ with a common boundary $S$ as follows:

$$
\begin{gathered}
Y_{1}=\left(\left(0, h_{1}\right) \cup\left(h_{2}, 1\right)\right) \times(0,1)^{2}, \quad Y_{2}=\left(h_{1}, h_{2}\right) \times(0,1)^{2}, \\
S=\left(\left\{h_{1}\right\} \cup\left\{h_{2}\right\}\right) \times(0,1)^{2} .
\end{gathered}
$$

It is obvious that $Y_{\varepsilon}=\varepsilon Y_{1} \cup \varepsilon Y_{2} \cup \varepsilon S$. The part $\varepsilon Y_{1}$ represents the first phase and consists of two layers with the same thickness $\varepsilon(1-h) / 2$ while the part $\varepsilon Y_{2}$ represents the second phase of the layered material and consists of one layer with the thickness $\varepsilon h$ (see Fig. 1).

The viscoelastic material we propose to study is described by the fractional Kelvin-Voigt model. Its constitutive equations between the components of the stress and strain tensors have the form

$$
\sigma_{i j}^{\varepsilon}=a_{i j k h}^{\varepsilon}(x) e_{k h}\left(u^{\varepsilon}\right)+b_{i j k h}^{\varepsilon}(x) e_{k h}\left(D_{t}^{\alpha} u^{\varepsilon}\right), \quad 0<\alpha<1,
$$

where $u^{\varepsilon}(x, t)$ is the displacement vector, $a^{\varepsilon}(x)=a\left(\varepsilon^{-1} x\right)$ and $b^{\varepsilon}(x)=b\left(\varepsilon^{-1} x\right)$ are $Y_{\varepsilon}$-periodic tensors describing the elastic and viscous properties of the material, $\sigma^{\varepsilon}$ and $e\left(u^{\varepsilon}\right)$ are the stress and strain tensors, and $D_{t}^{\alpha}$ is the Caputo fractional time derivative of order $\alpha$,

$$
\begin{gathered}
e_{k h}\left(u^{\varepsilon}\right)=\frac{1}{2}\left(\frac{\partial u_{k}^{\varepsilon}}{\partial x_{h}}+\frac{\partial u_{h}^{\varepsilon}}{\partial x_{k}}\right), \quad D_{t}^{\alpha} u^{\varepsilon}=\frac{1}{\Gamma(1-\alpha)} \int_{0}^{t}(t-\tau)^{-\alpha} \frac{\partial u^{\varepsilon}}{\partial \tau} d \tau, \\
a_{i j k h}(y)=\lambda_{s} \delta_{i j} \delta_{k h}+\mu_{s}\left(\delta_{i k} \delta_{j h}+\delta_{i h} \delta_{j k}\right), \quad y \in Y_{s}, \\
b_{i j k h}(y)=\zeta_{s} \delta_{i j} \delta_{k h}+\eta_{s}\left(\delta_{i k} \delta_{j h}+\delta_{i h} \delta_{j k}\right), \quad y \in Y_{s}, \\
y=\varepsilon^{-1} x, \quad s=1,2, \quad 1 \leqslant i, j, k, h \leqslant 3 .
\end{gathered}
$$




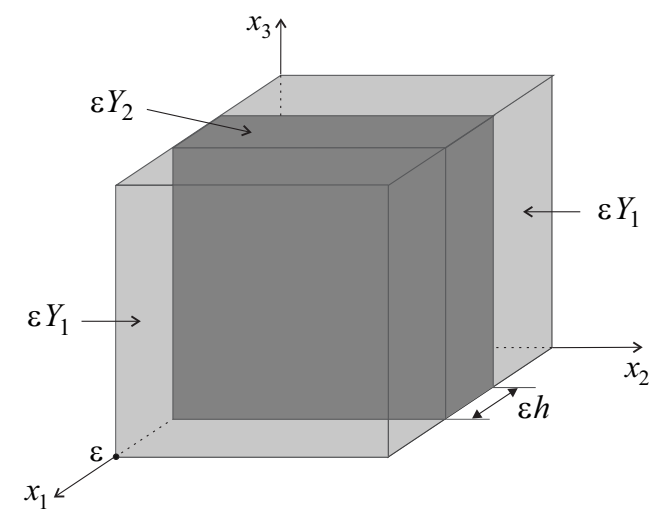

Fig. 1. The first and the second phases of the layered material

Here $\lambda_{s}$ and $\mu_{s}$ are the Lamé parameters of $\Omega_{s \varepsilon}, \zeta_{s}$ and $\eta_{s}$ are parameters describing the viscous behavior of $\Omega_{s \varepsilon}, \Gamma(\alpha)$ is Euler's gamma function, and $\delta_{i j}$ is Kroneker's delta. Note that in (1) and everywhere below we assume summation with respect to repeated indices.

The motion of the viscoelastic material in the phase $\Omega_{s \varepsilon}$ is described by the system of partial differential equations with fractional time derivative

$$
\rho_{s} \frac{\partial^{2} u_{i}^{\varepsilon}}{\partial t^{2}}=\frac{\partial \sigma_{i j}^{\varepsilon}}{\partial x_{j}}+f_{i}(x, t) \text { in } \Omega_{s \varepsilon} \times(0, T), \quad s=1,2,
$$

where $\rho_{s}=$ const $>0$ is the density of the material in $\Omega_{s \varepsilon}$ and $f_{i}(x, t)$ are the components of the volume external force vector.

On the boundaries between the layers we assume the condition of ideal contact. It means the continuity of displacements and normal stresses at each layer interface and is written as

$$
\left.\left[u^{\varepsilon}\right]\right|_{S_{\varepsilon}}=0,\left.\quad\left[\sigma_{i 1}^{\varepsilon}\right]\right|_{S_{\varepsilon}}=0,
$$

where the square brackets $\left.[\cdot]\right|_{S_{\varepsilon}}$ denote the jump in the enclosed quantity across the boundary $S_{\varepsilon}=\partial \Omega_{1 \varepsilon} \cap \partial \Omega_{2 \varepsilon}$.

Finally, we accept that the boundary conditions on $\partial \Omega$ for displacements as well as the initial conditions for displacements and velocities are homogeneous, i.e.

$$
\left.u^{\varepsilon}\right|_{\partial \Omega}=0,\left.\quad u^{\varepsilon}\right|_{t=0}=0,\left.\quad \frac{\partial u^{\varepsilon}}{\partial t}\right|_{t=0}=0 .
$$

Problem (2)-(4) is a mathematical model describing the general motion of the two-phase viscoelastic material. Our aim now is to deduce the corresponding effective (homogenized) model that describes the limit dynamic behavior of the original two-phase viscoelastic material as $\varepsilon \rightarrow 0$.

\section{Effective acoustic equations}

To construct the homogenized problem, we will use the method proposed in $[5,11,12]$. This method was developed for the homogenization of acoustics equations in two-phase dissipative media with periodic microstructure. Its main tools are the Laplace transform and the concept of two-scale convergence introduced by G. Nguetseng [9]. 
First, applying the Laplace transform $u^{\varepsilon}(x, t) \rightarrow u_{\lambda}^{\varepsilon}(x)$ and $f(x, t) \rightarrow f_{\lambda}(x)$, we convert the evolutionary problem (2)-(4) into the stationary one. As a result, we obtain the following boundary value problem for Laplace transforms:

$$
\begin{gathered}
\rho_{s} \lambda^{2} u_{\lambda i}^{\varepsilon}=\frac{\partial \sigma_{i j}^{\lambda \varepsilon}}{\partial x_{j}}+f_{\lambda i}(x) \text { in } \Omega_{s \varepsilon}, \quad s=1,2, \\
\left.u_{\lambda}^{\varepsilon}\right|_{\partial \Omega}=0,\left.\quad\left[u_{\lambda}^{\varepsilon}\right]\right|_{S_{\varepsilon}}=0,\left.\quad\left[\sigma_{i 1}^{\lambda \varepsilon}\right]\right|_{S_{\varepsilon}}=0
\end{gathered}
$$

where

$$
\sigma_{i j}^{\lambda \varepsilon}=\left(a_{i j k h}^{\varepsilon}(x)+\lambda^{\alpha} b_{i j k h}^{\varepsilon}(x)\right) e_{k h}\left(u_{\lambda}^{\varepsilon}\right)
$$

Next, using the basic properties of two-scale convergence and repeating the same arguments as in $[5,11,12]$, we can show that the homogenized problem that corresponds to problem (5) and which is constructed for $\varepsilon \rightarrow 0$ has the form

$$
\rho_{0} \lambda^{2} u_{\lambda i}=\frac{\partial \sigma_{i j}^{\lambda}}{\partial x_{j}}+f_{\lambda i}(x) \text { in } \Omega,\left.\quad u_{\lambda}\right|_{\partial \Omega}=0
$$

where

$$
\begin{gathered}
\rho_{0}=\rho_{1}(1-h)+\rho_{2} h, \quad \sigma_{i j}^{\lambda}=d_{i j k h}^{\lambda} e_{k h}\left(u_{\lambda}\right), \\
d_{i j k h}^{\lambda}=\int_{Y}\left(c_{i j k h}^{\lambda}(y)+c_{i j l m}^{\lambda}(y) e_{l m}^{y}\left(Q_{\lambda}^{k h}\right)\right) d y \\
c_{i j k h}^{\lambda}(y)=a_{i j k h}(y)+\lambda^{\alpha} b_{i j k h}(y), \quad e_{l m}^{y}\left(Q_{\lambda}^{k h}\right)=\frac{1}{2}\left(\frac{\partial Q_{\lambda l}^{k h}}{\partial y_{m}}+\frac{\partial Q_{\lambda m}^{k h}}{\partial y_{l}}\right) .
\end{gathered}
$$

Here the vector-valued functions $Q_{\lambda}^{k h}(y)$ are $Y$-periodic solutions to the following cell problems:

$$
\begin{gathered}
\frac{\partial}{\partial y_{j}}\left(c_{i j k h}^{\lambda}(y)+c_{i j l m}^{\lambda}(y) e_{l m}^{y}\left(Q_{\lambda}^{k h}\right)\right)=0 \text { in } Y, \quad \int_{Y} Q_{\lambda}^{k h} d y=0, \\
{\left.\left[Q_{\lambda}^{k h}\right]\right|_{y_{1}=h_{s}}=0,\left.\quad\left[c_{i j k h}^{\lambda}(y)+c_{i j l m}^{\lambda}(y) e_{l m}^{y}\left(Q_{\lambda}^{k h}\right)\right]\right|_{y_{1}=h_{s}}=0, \quad s=1,2 .}
\end{gathered}
$$

Now we apply the inverse Laplace transform to the homogenized stationary problem (6). We have

with

$$
\begin{aligned}
\rho_{0} \frac{\partial^{2} u_{i}}{\partial t^{2}} & =\frac{\partial \sigma_{i j}}{\partial x_{j}}+f_{i}(x, t) \quad \text { in } \Omega \times(0, T), \\
\left.u\right|_{\partial \Omega} & =0,\left.\quad u\right|_{t=0}=0,\left.\quad \frac{\partial u}{\partial t}\right|_{t=0}=0
\end{aligned}
$$

$$
\sigma_{i j}=d_{i j k h}(t) * e_{k h}(u)
$$

where the symbol $*$ denotes the operation of convolution with respect to time $t$.

\section{Solutions of auxiliary cell problems}

Passing to the inverse Laplace transforms in (8) we see that $Q^{k h}(y, t)$ depends on the Dirac function $\delta(t)$ and cannot be expressed in explicit form without some additional explanations. In order to do this and at the same time derive direct formula for calculation of components of 
the tensor $d(t)$, we will proceed in the following way. Let us represent the solutions $Q_{\lambda}^{k h}(y)$ to problems (8) in the form

$$
Q_{\lambda}^{k h}(y)=Z^{k h}(y)+\frac{V^{k h}(y)}{\lambda^{\alpha}-M^{k h}}, \quad M^{k h}=\text { const }
$$

where vector-valued functions $Z^{k h}(y), V^{k h}(y)$ and parameters $M^{k h}$ are to be specified.

In a first step, let us define the vector-valued functions $Z^{k h}(y)$ as $Y$-periodic solutions to the cell problems

$$
\begin{gathered}
\frac{\partial}{\partial y_{j}}\left(b_{i j k h}(y)+b_{i j l m}(y) e_{l m}^{y}\left(Z^{k h}\right)\right)=0 \text { in } Y, \quad \int_{Y} Z^{k h} d y=0, \\
{\left.\left[Z^{k h}\right]\right|_{y_{1}=h_{s}}=0,\left.\quad\left[b_{i j k h}(y)+b_{i j l m}(y) e_{l m}^{y}\left(Z^{k h}\right)\right]\right|_{y_{1}=h_{s}}=0, \quad s=1,2 .}
\end{gathered}
$$

In a second step, using the solutions $Z^{k h}(y)$ to problems (12), we define the vector-valued functions $V^{k h}(y)$ as $Y$-periodic solutions to the cell problems

$$
\begin{gathered}
\frac{\partial}{\partial y_{j}}\left(a_{i j k h}(y)+a_{i j l m}(y) e_{l m}^{y}\left(Z^{k h}\right)+b_{i j l m}(y) e_{l m}^{y}\left(V^{k h}\right)\right)=0 \quad \text { in } Y, \\
{\left.\left[a_{i j k h}(y)+a_{i j l m}(y) e_{l m}^{y}\left(Z^{k h}\right)+b_{i j l m}(y) e_{l m}^{y}\left(V^{k h}\right)\right]\right|_{y_{1}=h_{s}}=0, \quad s=1,2,} \\
\int_{Y} V^{k h} d y=0,\left.\quad\left[V^{k h}\right]\right|_{y_{1}=h_{s}}=0 .
\end{gathered}
$$

To write out solutions to problems (12) and (13), we introduce 1-periodic piecewise linear function $z\left(y_{1}\right)$ defined by

$$
z\left(y_{1}\right)= \begin{cases}\frac{y_{1} h}{1-h}, & y_{1} \in\left(0, h_{1}\right), \\ -y_{1}+\frac{1}{2}, & y_{1} \in\left(h_{1}, h_{2}\right), \\ \frac{\left(y_{1}-1\right) h}{1-h}, & y_{1} \in\left(h_{2}, 1\right) .\end{cases}
$$

It is easy to check that $Z^{k h}(y)=Z^{h k}(y)$ and $V^{k h}(y)=V^{h k}(y)$, so that we need only to find $Z^{k h}(y)$ and $V^{k h}(y)$ for $k \leqslant h$. Solving problems (12) for $k \leqslant h$, we obtain

$$
\begin{gathered}
Z^{11}(y)=\left(c_{1} z\left(y_{1}\right), 0,0\right), \quad Z^{22}(y)=Z^{33}(y)=\left(c_{2} z\left(y_{1}\right), 0,0\right), \\
Z^{12}(y)=\left(0, c_{3} z\left(y_{1}\right), 0\right), \quad Z^{13}(y)=\left(0,0, c_{3} z\left(y_{1}\right)\right), \quad Z^{23}(y)=(0,0,0),
\end{gathered}
$$

where

$$
\begin{gathered}
c_{1}=\frac{1}{b_{12}}(1-h)\left(b_{2}-b_{1}\right), \quad c_{2}=\frac{1}{b_{12}}(1-h)\left(\zeta_{2}-\zeta_{1}\right), \quad c_{3}=\frac{1}{\eta_{12}}(1-h)\left(\eta_{2}-\eta_{1}\right), \\
b_{12}=b_{1} h+b_{2}(1-h), \quad \eta_{12}=\eta_{1} h+\eta_{2}(1-h), \quad b_{s}=\zeta_{s}+2 \eta_{s}
\end{gathered}
$$

Substituting $Z^{k h}(y)$ into problems (13) and solving them for $k \leqslant h$, we derive

$$
\begin{gathered}
V^{11}(y)=\left(c_{4} z\left(y_{1}\right), 0,0\right), \quad V^{22}(y)=V^{33}(y)=\left(c_{5} z\left(y_{1}\right), 0,0\right), \\
V^{12}(y)=\left(0, c_{6} z\left(y_{1}\right), 0\right), \quad V^{13}(y)=\left(0,0, c_{6} z\left(y_{1}\right)\right), \quad V^{23}(y)=(0,0,0),
\end{gathered}
$$


where

$$
\begin{gathered}
c_{4}=\frac{1}{b_{12}^{2}}(1-h)\left(b_{1} a_{2}-b_{2} a_{1}\right), \quad c_{5}=\frac{1-h}{b_{12}^{2}}\left(\left(\lambda_{2}-\lambda_{1}\right) b_{12}-\left(\zeta_{2}-\zeta_{1}\right) a_{12}\right), \\
c_{6}=\frac{1}{\eta_{12}^{2}}(1-h)\left(\eta_{1} \mu_{2}-\eta_{2} \mu_{1}\right), \quad a_{12}=a_{1} h+a_{2}(1-h), \quad a_{s}=\lambda_{s}+2 \mu_{s} .
\end{gathered}
$$

Now, after defining $Z^{k h}(y)$ and $V^{k h}(y)$ in (11), we can find parameters $M^{k h}$. It follows from (8), (12), and (13) that $M^{k h}$ satisfies the system

$$
\begin{gathered}
\frac{\partial}{\partial y_{j}}\left(a_{i j l m}(y) e_{l m}\left(V^{k h}\right)+M^{k h} b_{i j l m}(y) e_{l m}^{y}\left(V^{k h}\right)\right)=0 \quad \text { in } Y, \\
{\left.\left[a_{i j l m}(y) e_{l m}\left(V^{k h}\right)+M^{k h} b_{i j l m}(y) e_{l m}^{y}\left(V^{k h}\right)\right]\right|_{y_{1}=h_{s}}=0, \quad s=1,2 .}
\end{gathered}
$$

Substitute $V^{k h}(y)$ found above into (14) and (15). It is easy to check that equations (14) are always fulfilled for any parameters $M^{k h}$. Further, from the boundary conditions (15) we calculate the required values of $M^{k h}$ :

$$
\begin{gathered}
M^{11}=M^{22}=M^{33}=-\frac{a_{12}}{b_{12}}, \\
M^{12}=M^{21}=M^{13}=M^{31}=-\frac{\mu_{12}}{\eta_{12}} .
\end{gathered}
$$

Applying the inverse Laplace transform to (11), we get

$$
Q^{k h}(y, t)=\delta(t) Z^{k h}(y)+R_{\alpha-1}\left(M^{k h}, t\right) V^{k h}(y),
$$

where $R_{\nu}(\beta, t)$ denotes fractional exponential Rabotnov's function [13]:

$$
R_{\nu}(\beta, t)=t^{\nu} \sum_{n=0}^{\infty} \frac{\beta^{n} t^{n(1+\nu)}}{\Gamma[(1+n)(1+\nu)]} .
$$

Next we substitute the decomposition (11) into (7) to obtain

$$
d_{i j k h}^{\lambda}=A_{i j k h}+\lambda^{\alpha} B_{i j k h}+G_{i j k h}(\lambda),
$$

where the components of the tensors $A, B$, and $G(\lambda)$ are given by the formulas

$$
\begin{gathered}
A_{i j k h}=\int_{Y}\left(a_{i j k h}(y)+a_{i j l m}(y) e_{l m}^{y}\left(Z^{k h}\right)+b_{i j l m}(y) e_{l m}^{y}\left(V^{k h}\right)\right) d y, \\
B_{i j k h}=\int_{Y}\left(b_{i j k h}(y)+b_{i j l m}(y) e_{l m}^{y}\left(Z^{k h}\right)\right) d y, \\
G_{i j k h}(\lambda)=\frac{1}{\lambda^{\alpha}-M^{k h}} \int_{Y}\left(a_{i j l m}(y) e_{l m}^{y}\left(V^{k h}\right)+M^{k h} b_{i j l m}(y) e_{l m}^{y}\left(V^{k h}\right)\right) d y .
\end{gathered}
$$

Therefore, the constitutive equations (10) take the form

$$
\sigma_{i j}=A_{i j k h} e_{k h}(u)+B_{i j k h} e_{k h}\left(D_{t}^{\alpha} u\right)+G_{i j k h}(t) * e_{k h}(u),
$$

where $G_{i j k h}(t)$ are the inverse Laplace transforms of $G_{i j k h}(\lambda)$ :

$$
G_{i j k h}(t)=R_{\alpha-1}\left(M^{k h}, t\right) \int_{Y}\left(a_{i j l m}(y) e_{l m}\left(V^{k h}\right)+M^{k h} b_{i j l m}(y) e_{l m}^{y}\left(V^{k h}\right)\right) d y .
$$

From (18) we see that the effective acoustic equations (9) are partial integro-differential equations with fractional time derivative and constant coefficients. It is interesting to note that their kernels are expressed via two different Rabotnov's functions $R_{\alpha-1}\left(-a_{12} / b_{12}, t\right)$ and $R_{\alpha-1}\left(-\mu_{12} / \eta_{12}, t\right)$. 


\section{Components of the tensors $A, B$, and $G(t)$}

Before proceeding to the calculation of the tensors $A, B$, and $G(t)$, let us note that

$$
\begin{gathered}
A_{i j k h}=A_{j i k h}=A_{k h i j}, \quad A_{i j k h}=0 \quad \text { whenever } \delta_{i j} \delta_{k h}+\delta_{i k} \delta_{j h}+\delta_{i h} \delta_{j k}=0, \\
A_{2222}=A_{3333}, \quad A_{1122}=A_{1133}, \quad A_{1212}=A_{1313}, \quad A_{2222}-A_{2233}=2 A_{2323}
\end{gathered}
$$

and similarly for the tensors $B$ and $G(t)$. Moreover, it is easy to see that

$$
A_{2323}=\mu_{1}(1-h)+\mu_{2} h, \quad B_{2323}=\eta_{1}(1-h)+\eta_{2} h, \quad G_{2323}(t)=0 .
$$

Therefore, it is sufficient to find the components of $A, B$, and $G(t)$ with indexes $\{1111\}$, $\{2222\},\{1122\}$, and $\{1212\}$. To do this, we first substitute the found solutions to problems (12) and (13) into formulas (16) and (17). This yeilds

$$
\begin{gathered}
A_{1111}=a_{1}(1-h)+a_{2} h+c_{1} h\left(a_{1}-a_{2}\right)+c_{4} h\left(b_{1}-b_{2}\right), \\
A_{2222}=a_{1}(1-h)+a_{2} h+c_{2} h\left(\lambda_{1}-\lambda_{2}\right)+c_{5} h\left(\zeta_{1}-\zeta_{2}\right), \\
A_{1122}=\lambda_{1}(1-h)+\lambda_{2} h+c_{2} h\left(a_{1}-a_{2}\right)+c_{5} h\left(b_{1}-b_{2}\right), \\
A_{1212}=\mu_{1}(1-h)+\mu_{2} h+c_{3} h\left(\mu_{1}-\mu_{2}\right)+c_{6} h\left(\eta_{1}-\eta_{2}\right), \\
B_{1111}=b_{1}(1-h)+b_{2} h+c_{1} h\left(b_{1}-b_{2}\right), \quad B_{2222}=b_{1}(1-h)+b_{2} h+c_{2} h\left(\zeta_{1}-\zeta_{2}\right), \\
B_{1212}=\eta_{1}(1-h)+\eta_{2} h+c_{3} h\left(\eta_{1}-\eta_{2}\right), \quad B_{1122}=\zeta_{1}(1-h)+\zeta_{2} h+c_{2} h\left(b_{1}-b_{2}\right) .
\end{gathered}
$$

Taking into account the above values of constants $c_{i}$ and using trivial transformations, we obtain

$$
\begin{gathered}
A_{1111}=\frac{1}{b_{12}^{2}}\left(a_{2} b_{1}^{2} h+a_{1} b_{2}^{2}(1-h)\right), \quad A_{1212}=\frac{1}{\eta_{12}^{2}}\left(\mu_{2} \eta_{1}^{2} h+\mu_{1} \eta_{2}^{2}(1-h)\right), \\
A_{2222}=a_{1}(1-h)+a_{2} h+\frac{h(1-h)\left(\zeta_{1}-\zeta_{2}\right)}{b_{12}^{2}}\left(a_{12}\left(\zeta_{1}-\zeta_{2}\right)-2 b_{12}\left(\lambda_{1}-\lambda_{2}\right)\right), \\
A_{1122}=\frac{1}{b_{12}}\left(b_{1} \lambda_{2} h+b_{2} \lambda_{1}(1-h)\right)+\frac{h}{b_{12}^{2}}(1-h)\left(\zeta_{2}-\zeta_{1}\right)\left(a_{1} b_{2}-a_{2} b_{1}\right), \\
B_{1111}=\frac{b_{1} b_{2}}{b_{12}}, \quad B_{2222}=b_{1}(1-h)+b_{2} h-\frac{h}{b_{12}}(1-h)\left(\zeta_{1}-\zeta_{2}\right)^{2}, \\
B_{1122}=\frac{1}{b_{12}}\left(b_{1} \zeta_{2} h+b_{2} \zeta_{1}(1-h)\right), \quad B_{1212}=\frac{\eta_{1} \eta_{2}}{\eta_{12}} .
\end{gathered}
$$

In order to find the components of $G(t)$, we substitute the solutions $V^{k h}(y)$ to problems (13) and the parameters $M^{k h}$ into formulas (19). As a result, we get

$$
\begin{gathered}
G_{1111}(t)=-\frac{h(1-h)}{b_{12}^{3}}\left(a_{1} b_{2}-a_{2} b_{1}\right)^{2} R_{\alpha-1}\left(-\frac{a_{12}}{b_{12}}, t\right) \\
G_{2222}(t)=-\frac{h(1-h)}{b_{12}^{3}}\left(\left(\lambda_{1}-\lambda_{2}\right) b_{12}-\left(\zeta_{1}-\zeta_{2}\right) a_{12}\right)^{2} R_{\alpha-1}\left(-\frac{a_{12}}{b_{12}}, t\right), \\
G_{1122}(t)=-\frac{h(1-h)}{b_{12}^{3}}\left(a_{1} b_{2}-a_{2} b_{1}\right)\left(\left(\lambda_{1}-\lambda_{2}\right) b_{12}-\left(\zeta_{1}-\zeta_{2}\right) a_{12}\right) R_{\alpha-1}\left(-\frac{a_{12}}{b_{12}}, t\right), \\
G_{1212}(t)=-\frac{h(1-h)}{\eta_{12}^{3}}\left(\mu_{1} \eta_{2}-\mu_{2} \eta_{1}\right)^{2} R_{\alpha-1}\left(-\frac{\mu_{12}}{\eta_{12}}, t\right) .
\end{gathered}
$$


To conclude, we note that our results can be considered as a generalization of those obtained in the case of two-phase layered viscoelastic material described by a standard Kelvin-Voigt model $(\alpha=1)$. Indeed, the effective acoustic equations for the last material also have form (9) with the constitutive equations (18), where $A_{i j k h}$ and $B_{i j k h}$ are defined by the same formulas as above. Moreover, the components of $G(t)$ are found by using the formulas presented here, in which we should put $\alpha=1$ and take into account that

$$
R_{0}\left(-\frac{a_{12}}{b_{12}}, t\right)=\exp \left(-\frac{a_{12} t}{b_{12}}\right), \quad R_{0}\left(-\frac{\mu_{12}}{\eta_{12}}, t\right)=\exp \left(-\frac{\mu_{12} t}{\eta_{12}}\right) .
$$

This work was accomplished within the Russian State Assignment under contract no. AAAAA20-120011690138-6.

\section{References}

[1] E.Sanchez-Palencia, Non-Homogeneous Media and Vibration Theory, Lecture Notes in Physics, Vol. 127, Springer, 1980.

[2] Z.Abdessamad, I.Kostin, G.Panasenko, V.P.Smyshlyaev, Memory effect in homogenization of a viscoelastic Kelvin-Voigt model with time-dependent coefficients, Math. Meth. Appl. Sci., 19(2009), no. 9, 1603-1630.

[3] R.P.Gilbert, A.Panchenko, X.Xie, Homogenization of a viscoelastic matrix in linear frictional contact, Math. Meth. Appl. Sci., 28(2005), 309-328.

[4] H.I.Ene, M.L.Mascarenhas, J.Saint Jean Paulin, Fading memory effects in elasticviscoelastic composites, Math. Model. Numer. Anal., 31(1997), no. 7, 927-952.

[5] A.S.Shamaev, V.V.Shumilova, On the spectrum of one-dimensional vibrations in a medium consisting of elastic and viscoelastic Kelvin-Voigt materials, Zh. Vychisl. Mat. Mat. Fiz., 53(2013), no. 2, 282-290 (in Russian). DOI: 10.7868/S0044466913020142

[6] F.Mainardi, Fractional Calculus and Waves in Linear Viscoelasticity: An Introduction to Mathematical Models, Imperial College Press, London, 2010.

[7] Yu.A.Rossikhin, M.V.Shitikova, Application of fractional calculus for dynamic problems of solid mechanics: novel trends and recent results, Appl. Mech. Rev., 63(2010), no. 1, 010801/1-52. DOI: $10.1115 / 1.4000563$

[8] T.M.Atanacković, S.Pilipović, B.Stanković, D.Zorica, Fractional Calculus With Applications in Mechanics: Wave Propagation, Impact and Variational Principles, Wiley-ISTE, London, Hoboken, 2014.

[9] G.Nguetseng, A general convergence result for a functional related to the theory of homogenization, SIAM J. Math. Anal. 20(1989), no. 3, 608-623.

[10] G.Allaire, Homogenization and two-scale convergence, SIAM J. Math. Anal., 23(1992), no. $6,1482-1518$.

[11] A.S.Shamaev, V.V.Shumilova, On the spectrum of one-dimensional oscillations of a laminated composite with components of elastic and viscoelastic materials, Sib. Zhurnal Indust. Matematiki, 15(2012), no. 4, 124-134 (in Russian). 
[12] V.V.Shumilova, Homogenizing the viscoelasticity problem with long-term memory, Math. Notes, 94(2013), no. 3, 414-425. DOI: 10.1134/S0001434613090125

[13] Yu.N.Rabotnov, Elementy nasledstvennoy mekhaniki tverdykh tel, Nauka, Moscow, 1977 (in Russian).

\title{
Эффективные уравнения акустики для слоистого материала, описываемого дробной моделью Кельвина-Фойгта
}

\author{
Алексей С. Шамаев \\ Владлена В. Шумилова \\ Институт проблем механики им. А. Ю. Ишлинского РАН \\ Москва, Российская Федерация
}

\begin{abstract}
Аннотация. Статья посвящена построению эффективных уравнений акустики для двухфазного слоистого вязкоупругого материала, описываемого моделью Кельвина-Фойгта с дробными производными по времени. Для этой цели используется теория двухмасштабной сходимости и преобразование Лапласа по времени. Показано, что эффективные уравнения являются интегродифференциальными уравнениями в частных производных с дробными производными по времени и дробно-экспоненциальными ядрами свертки. Для того чтобы найти коэффициенты и ядра сверток этих уравнений, сформулированы и решены несколько вспомогательных задач.
\end{abstract}

Ключевые слова: усреднение, уравнения акустики, вязкоупругость, дробная модель КельвинаФойгта. 